\title{
Global Environmental Assessment Requires Global Functional Searching Engines: Robust Application of TaToo Tools
}

\author{
Miroslav Kubásek ${ }^{1}$, Jiří Hřebíček ${ }^{1}$, Jiří Kalina ${ }^{1}$, Ladislav Dušek ${ }^{1}$, \\ Jaroslav Urbánek ${ }^{2}$, and Ivan Holoubek ${ }^{2}$ \\ ${ }^{1}$ IBA, Masaryk University, Kamenice 126/3, 62500 Brno, Czech Republic \\ ${ }^{2}$ RECETOX, Masaryk University, Kamenice 126/3, 62500 Brno, Czech Republic \\ \{kubasek, hrebicek, kalina, dusek\}@iba.muni.cz, \\ \{urbanek, holoubek\} @recetox.muni.cz
}

\begin{abstract}
The synthesis of existing Persistent Organic Pollutants (POPs) pollution monitoring databases with epidemiological data is considered for identifying some impacts of POPs on human health. This task requires new, rich, data, services and models discovery capabilities from a multitude of monitoring networks and web resources. The FP7 project TaToo (Tagging Tool based on a Semantic Discovery Framework) is setting up a semantic web solution to close the discovery gap that prevents a full and easy access to web resources. The use of TaToo tools together with software GENASIS and SVOD is discussed as TaToo validation scenario for anthropogenic impact and global climate change influence on POPs trajectory.
\end{abstract}

Keywords: TaToo, semantic web, POPs, Stockholm Convention, GENASIS, SVOD, anthropogenic impact, global climate change.

\section{Introduction}

The FP7 project TaToo (Tagging Tool based on a Semantic Discovery Framework) aims to set up a semantic web solution to close the discovery gap that prevents a full and easy access to environmental resources on the web [8]. The core of the project will focus on the development of tools allowing third parties to easily discover environmental resources (data and/or services residing on different information nodes) on the web and to add valuable information in the form of semantic annotations to these resources, thus facilitating future usage and discovery, and kicking off a beneficial cycle of information enrichment.

TaToo validates the usability of its developments through the implementation of three different scenarios. All three scenarios are embedded in highly complex environmental domains and are therefore mainly addressed to domain expert groups and communities as well as to technically skilled users.

This paper contains the detailed framework of Masaryk university scenario, which is the anthropogenic impact and the influence of global climate change on this impact [4], [5]. The purpose of the paper is to give the overview of the scenario (background, 
objectives, and available tools), the definition of users who will use the TaToo tools, describe the possible use cases, and to provide with the mock ups.

\section{Description of the Scenario}

The third validation scenario of TaToo project named Anthropogenic impact and global climate change is managed by Masaryk University ${ }^{1}$ (MU). MU validation scenario is dealing with the correlation of environmental pollutants and their health impact on the population and the correlation of transport of environmental pollutants with global climate change [4], [5]. The aim is to create a central place for researchers, domain experts and decision makers to discover and access interdisciplinary knowledge in more efficient and usable way that is the currently state of the art. Due to the fact that there is an enormous amount of information resources in scientific fields, which is steadily growing, available search mechanisms like search engines, scientific networks and similar technologies are not sufficient to meet the complex requirements of today's researchers and scientists. The result of conventional discovery processes are often not matching the domain context of the users and obligate them the tedious task of filtering large result sets to obtain the original object of the interest of the researcher intended to find with the search. Therefore the need arises for an improving discovery method, which will incorporate the domain knowledge and additional semantic information into the search in order to obtain a more fitting result for the specific context of the user.

This validation scenario not only aims to improve the discovery of scientific resources for one particular domain, but also tries to discover and create new relationships among different domains. The correlation of environmental pollutants including their transport due to global climate change and their health impact on the population is only one significant example of creating new relationships among different domains. These dependencies could represent new scientific insights for already available resources and connect the knowledge of the single domains. These relationships should facilitate further discovery process to deliver matching resources of multiple domains.

This validation scenario represents the close cooperation and joint venture of two university institutes: the Research Centre for Toxic Compounds in the Environment ${ }^{2}$ (RECETOX) and Institute of Biostatistics and Analyses ${ }^{3}$ (IBA).

RECETOX is an independent institute of the Masaryk University. RECETOX performs research, development, education and expertise in the field of environmental contamination by toxic compounds with specific focus on persistent organic pollutants (POPs), polar organic compounds, toxic metals and their species and natural toxins - cyanotoxins. It is also Stockholm Convention Regional centre ${ }^{4}$ for capacity building and transfer of technology in Central and Eastern European countries. The Stockholm Convention on Persistent Organic Pollutants ${ }^{5}$ (Stockholm

\footnotetext{
${ }^{1}$ http://www.muni.cz

${ }^{2} \mathrm{http}: / /$ www.recetox.muni.cz

${ }^{3} \mathrm{http}: / /$ www.iba.muni.cz

${ }^{4} \mathrm{http}: / /$ www.recetox.muni.cz/index-en.php?pg=regional-pops-center

${ }^{5} \mathrm{http} / / /$ www.pops.int
} 
convention) is a global treaty to protect human health and the environment from chemicals that remain intact in the environment for long periods, become widely distributed geographically and accumulate in the fatty tissue of humans and wildlife. RECETOX is formed by several research divisions, service laboratories and technology-transfer centres: Environmental chemistry and modelling, Ecotoxicology and risk assessment, Trace laboratory, and Laboratory of data analyses. Research and development of the centre include monitoring of environmental matrices, studies of environmental fate and effects (ecotoxicology) of toxic compounds, ecological and human risk assessment as well as the development of informational and expert systems. In January 2010 RECETOX launched the first version of the Global Environmental Assessment and Information System (GENASIS), which provides information support for implementation of the Stockholm convention at an international level. The initial phase of the GENASIS project is focused on data from regular monitoring programmes of POPs, providing a general overview of spatial patterns and temporal trends of pollutants concentrations [1].

IBA is a research institute focused on delivering solutions to questions arising in scientific projects and providing related services, especially in the field of biological and clinical data analysis, organization and management of clinical trials, software development and Information and Communication Technology (ICT) applications. IBA activities are primarily focused on organizational and expert services for large scientific projects and clinical research projects. IBA is formed by four divisions: Division of Data Analysis, Division of Clinical Trials, Division of Information and Communication Technologies, and Division of Environmental Informatics and Modelling. For example, IBA created the web portal for epidemiology of malignant tumours in the Czech Republic, the System for Visualizing of Oncological Data $(\mathrm{SVOD})^{7}$, based on the data from the Czech National Oncology Registry [3].

Dušek [2] pointed in 2009:"A full-area monitoring of the environmental risk factors in all main environmental components is performed in the Czech Republic. The main objective of this functional monitoring network is the estimation of exposure to xenobiotic substances, and the evaluation of subsequent risks to human health. The system provides information for health risks management and also serves for public education, which is a prerequisite for active care of one's own health. The outputs from monitoring systems may also be used for assessing human risks associated with cancer epidemiology. Data about POPs are of key importance, since these compounds are known to have a wide spectrum of carcinogenic effects, a tendency to bioaccumulation, and are subject to long-distance transport."

The objective of the MU validation scenario is to use and validate the resulting tagging and discovery framework of the TaToo project. Since the primary scope of the TaToo project is to facilitate the discovery of environmental resources, this scenario delivers the perfect opportunity to validate the resulting solution against challenging real word problems. There are numerous scientific domains available and actively researched at the MU, but two important domains have been carefully chosen to demonstrate and validate the envisioned functionality of the TaToo project. The vision of the MU validation scenario is that other scientific domains could follow the

\footnotetext{
${ }^{6}$ http://www.genasis.cz

${ }^{7}$ http://www.svod.cz
} 
initial institutes to further spin a new kind of knowledge network to deliver a new generation of tools and methods to effectively and conveniently support the scientific user in their daily work.

\subsection{System for Visualizing of Oncological Data}

Creating the SVOD web portal about tumour epidemiology in the Czech Republic was primarily motivated by the effort to make this representative and valuable data available to wide spectrum of users. We anticipated that general epidemiology data about these serious diseases and related population risks should be freely available to everybody in the Czech Republic. Another ambition of the SVOD web portal was to provide relevant information about tumor epidemiology in the Czech Republic abroad.

The SVOD web portal information services can be divided into three sections:

1) Current news: regularly updated information about population risk assessment and tumor epidemiology;

2) Interactive analyses that allow the user to investigate directly epidemiological trends of selected oncological diagnoses;

3) Predefined presentations of important topics (Authorised information service).

These services are available free to all users. All analyses contain only safe and publicable data of tumour epidemiology, without any personal data of patients.

The project of creating the SVOD web portal for tumour epidemiology in the Czech Republic was tied with longstanding development of analytical software for data from National Oncology Registry (NOR) of the Czech Republic. The SVOD portal was created in the years 1999-2003. Currently is used its sixth version. The SVOD web portal solves all these problems and provides an effective way of access to epidemiological analyses to unlimited number of users. It makes accessible all NOR data via wide range of automated analyses. Although the SVOD was finalized successfully, there are severe limits with its distribution and availability, [3].

\subsection{Global Environmental Assessment Information System}

The GENASIS web portal provides information support for the implementation of the Stockholm Convention at the international level. This environmental information system was developed as a subsystem of the Single Information System of the Environment $^{8}$ (JISŽP) of the Ministry of Environment of the Czech Republic, which will be connected to Share Environmental Information System ${ }^{9}$ (SEIS) of European Union [7]. GENASIS connection with other accessible information sources creates the potential for a comprehensive assessment of anthropogenic impact on the environment and the associated ecological and health risks. The GENASIS portal contains data collected by RECETOX and its partners since 1988 in various monitoring types (long-term, short-term, research studies ...).

\footnotetext{
${ }^{8} \mathrm{http} / / /$ www.mzp.cz/cz/jednotny_informacni_system_zivotni_prostredi

${ }^{9} \mathrm{http} / / /$ www.eea.europa.eu/about-us/what/shared-environmental-information-system
} 
The GENASIS portal also offers analytical tools, one of the most important parts of the web portal. These tools allow basic processing of measured environmental data by "statistical" application programs. The user of GENASIS web portal can determine in the introductory screen what kind of data will enter to the analysis by the selection of various parameters (e.g. project name, sampling time, matrix, chemical compound, etc.). It will obtain by this way core set of data. The GENASIS system is able to visualize location of each sampled site where data are monitored or measured by the means of synoptic maps and examine general and / or detailed information about sampling frequency. It is also possible to sort and select / deselect monitored localities and view measured concentrations of selected compounds at these localities.

However, mere visualization is not the main objective of the development od analytical tools. Using additional GENASIS modules it is possible to obtain descriptive statistics for selected data set, observe changes in concentration of the user-selected chemicals during time period and easily depict seasonal and long-term trends (Fig. 1).

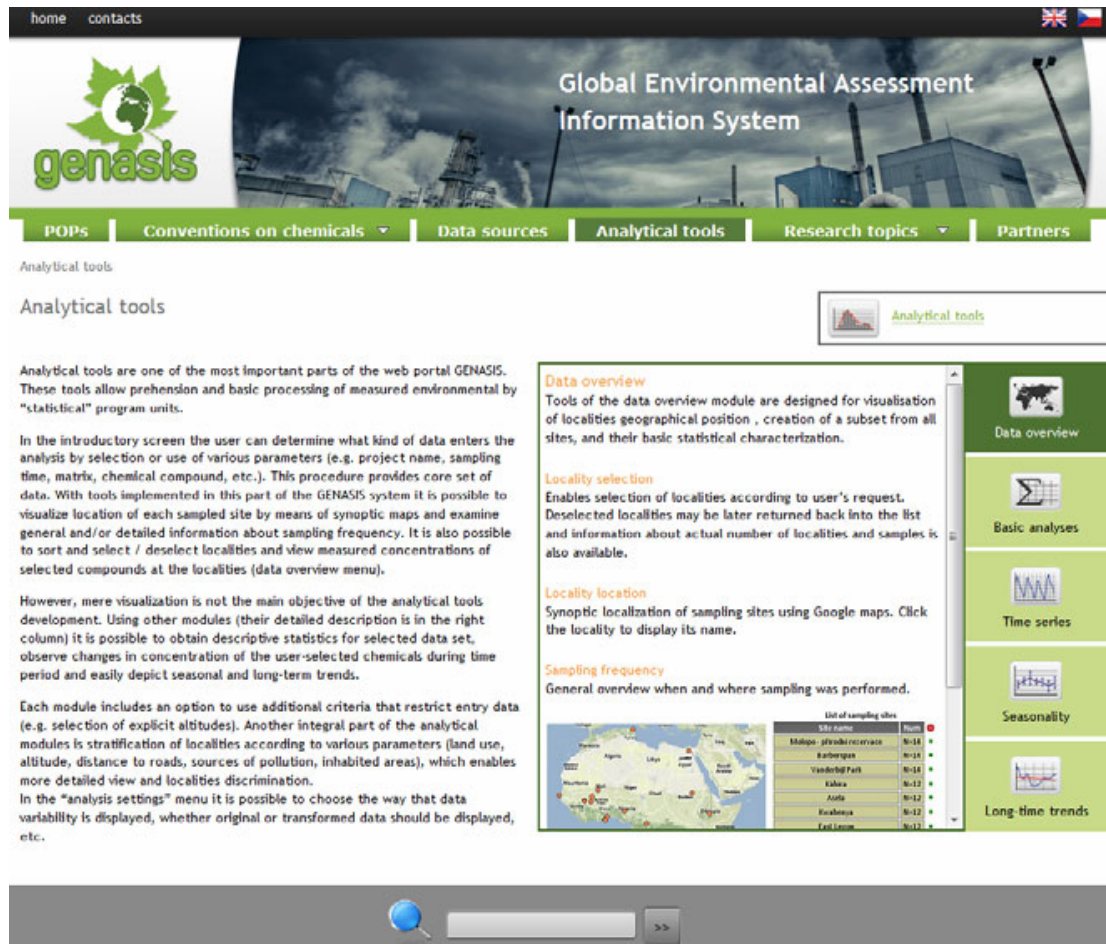

Fig. 1. GENASIS Analytical Tools

Each module includes an option to use additional criteria that restrict entry data (e.g. selection of explicit altitudes). Another integral part of the analytical modules is stratification of localities according to various parameters (land use, altitude, distance to roads, sources of pollution, inhabited areas), which enables more detailed view and 
localities discrimination. More complex analyses and models are currently and continuously being prepared.

The pilot version of GENASIS uses data from monitoring network MONET [6], [9], which is focused on occurrence of POPs in an ambient air of the Central and Eastern European region (CEEC), Central Asia, Africa and Pacific Islands driven by RECETOX as the Regional Center of the Stockholm Convention for the CEEC region. For many of the participating countries these activities generated first data on the atmospheric levels of POPs. But the primary motivation for GENASIS project is to make all representative and very valuable data about presence and distribution of these dangerous substances in the environment accessible for wide forum of users and interested public.

GENASIS project uses data collected both within the National Implementation Plan for the Implementation of the Stockholm Convention in the Czech Republic $(\mathrm{NIP})^{10}$ and international projects to reach its goals. The Czech Republic has had a long-term tradition in POPs monitoring in the environment and its monitoring networks cover all environmental components. A basic description supplemented by outputs used within the frame of the NIP is available for each monitoring network.

\section{Relevance of the Scenario}

The synthesis of existing (air) pollution monitoring databases (e.g. EEA Datasets ${ }^{11}$, AirBase $^{12}$ etc.), with epidemiological databases (e.g. Virtual Library: Medicine and Health: Epidemiology ${ }^{13}$, NOR etc.) is required for identifying the effects of pollution on human health (anthropogenic impact). This task requires new, rich, data discovery capabilities within the bodies of available knowledge. IBA and RECETOX customers pose requests for new anthropogenic impact studies and influence of global climate change (e.g. a contamination of all environmental components by POPs through their changed transport due to global climate change) requiring data discovery from a multitude of monitoring networks and resources. Proper use of such data requires contextual information, which TaToo will deliver through tagging and enhanced information description (meta-data) provided by an appropriate semantic environment. In this context, MU intends to employ TaToo tools and validate their performance for tagging and semantic rich discovery of anthropogenic impact and global climate change resources. TaToo aims to set up a semantic web solution to close the discovery gap that prevents a full and easy access to environmental resources on the web.

TaToo developments will be demonstrated in the domain of anthropogenic impact and global climate change analysis. To achieve such a goal, it is necessary to use data from national and international monitoring networks, and to discover and obtain ascomplete-as-possible data sets representing environmental anthropogenic impact.

\footnotetext{
${ }^{10} \mathrm{http} / / / \mathrm{www} . r e c e t o x . m u n i . c z / p o p s-c e n t r u m / i n d e x . p h p ? p g=p o p s--n i p$

${ }^{11} \mathrm{http} / / / \mathrm{www} . e e a . e u r o p a . e u /$ data-and-maps/data\#c5=all\&b_start=0\&c9=air\%2520emissions\&c11=air

${ }^{12} \mathrm{http} / / /$ acm.eionet.europa.eu/databases/airbase/index_html

${ }^{13} \mathrm{http} / / / \mathrm{www} . e$ ibiostat.ucsf.edu/epidem/epidem.html
} 
Discovery, use, and reuse of these data require enhancements of meta-information descriptions, which can be achieved through TaToo's semantic rich environment (Fig.2).

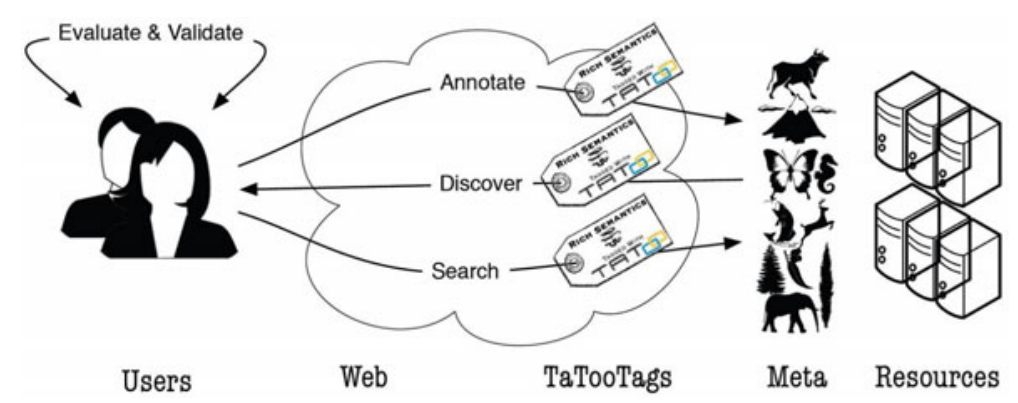

Fig. 2. TaToo's semantic rich environment of information resources

In this context, MU intends to employ TaToo tools and validate their performance for tagging and semantic rich discovery of resources of anthropogenic impact and the influence of global climate change on the transport of pollutants.

Climate is a factor strongly interacting with transport, transformation and effects of POPs in the environment. They are emitted into ambient air from various primary and secondary sources and atmosphere plays a key role in their transport both around their source surroundings and on long distances. Atmospheric transport is also main pathway of POPs transport into aquatic and terrestrial ecosystems. Current research of POPs global fate searches new information on sources, but also on other factors that affect pollutants concentration in ambient air, because climate, processes at the interface of air and soil or water surface, and atmospheric transport significantly affect spatial and time variability of POPs in ambient air. From this point of view, regular measurements of pollutants concentration in ambient air at various localities and monitoring studies at various levels from immediate vicinity of local point sources up to continental level are of key importance. Important components of these measurements are monitoring design, selection of monitored chemicals, selection of sampling and analytical methods, processing method, and data interpretation.

Scenarios of climate changes predict decreasing temperature contrast between poles and equator, drier continental interiors, wetter arctic and sub polar regions, modification of wind and precipitation patterns, sea level rise and others. All these environmental changes can influence the level of POPs in the environment, their partitioning among environmental compartments (air, soil, water), long-range transport, degradation rates, and toxic effects. Also, the release of POPs can be higher, for example due to pesticides usage to stop potential increase of malaria disease. Higher concentrations of POPs in the environment would then probably have more serious effects on living organisms.

The GENASIS information system is based on database and linked analytical tools providing information base available on the web portal [1]. Visualization of temporal and spatial patterns linked to characteristics of chemical compounds involved in 
Stockholm Convention support development of scenarios for individual environmental compartments.

Initial version of GENASIS system contains air pollution data and data from other matrices are being prepared. Analytical tools of GENASIS system provide visualization of this data and basic statistics. The distribution models of POPS transport will be implemented in the near future to predict the fate of POPs in the environment. The user of TaToo tools would be able to find and explore such models from GENASIS website and also other relevant resources to investigate effects on the fate of POPs caused by global climate change.

TaToo tools will be validated over specific scenarios and they will allow for continued collaborative development by federated users communities.
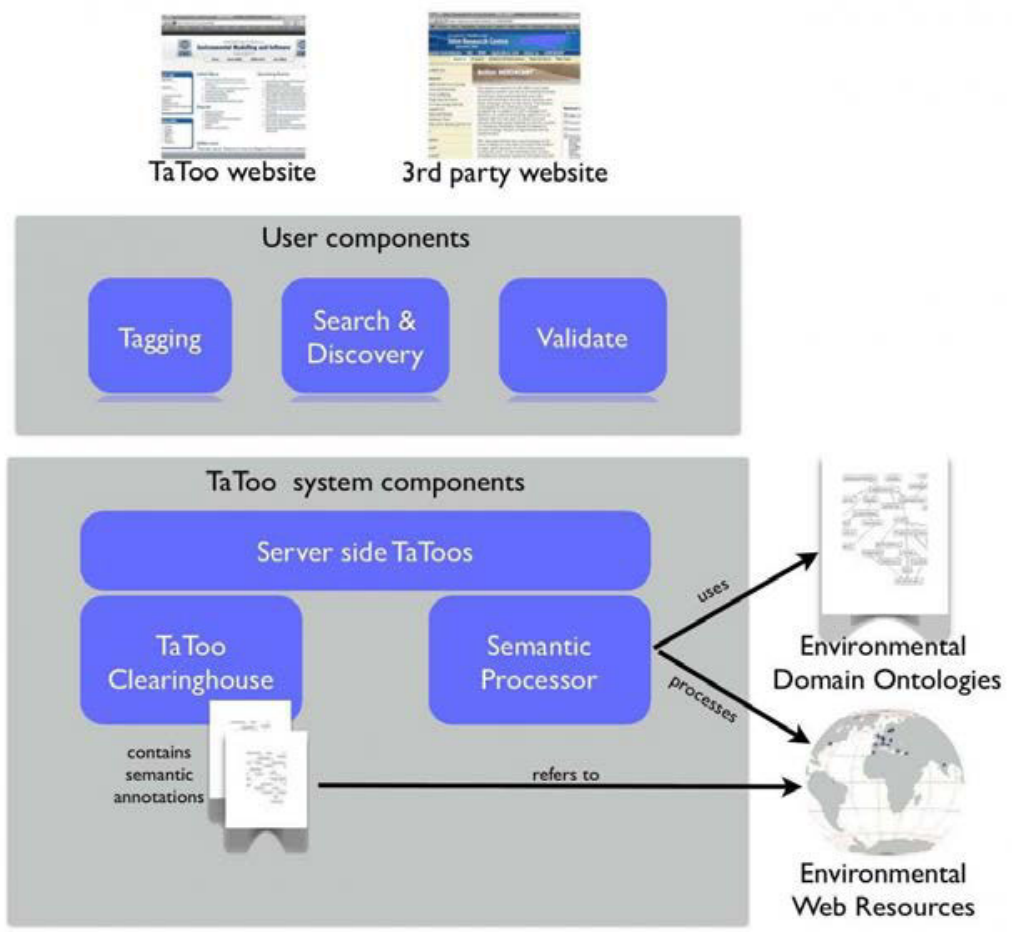

Fig. 3. TaToo architecture ${ }^{14}$

\section{TaToo Architecture}

TaToo aims to deliver a web based system centred on a three main elements:

- a clearinghouse, which plays the role of organising the semantic information on environmental resources. The clearinghouse contains a list of semantic

\footnotetext{
${ }^{14} \mathrm{http} / / /$ www.tatoo-fp7.eu/tatooweb/?q=node/20
} 
annotations of environmental resources, referring to the original content as available on the world wide web.

- $\quad$ a semantic processor, a core component of the TaToo system, since it uses a set of (pluggable) environmental ontologies to provide semantic services to the tagging tools of TaToo.

- $\quad$ a set of tagging tools (TaToos), which offer services such as tagging new environmental resources, including quality and uncertainty information, searching for and discovering tagged environmental resources, validating the results of a search.

The server side interacts with web based client components. These components can be interactively combined in order to enable human users to create, modify, delete and update TaToos. The composition is open, so that third party will be able to exploit the components to deliver their specifically targeted services.

The TaToo Web Portal represents the main entry point for the TaToo Semantic Framework and its functionality.

Due to the nature of a Web Portal the user has access independently of his location to all the Tagging, Discovery and Evaluation/Validation functionality. The TaToo Web Portal implements user first level authentication and authorization for access control and offers a customisable portal content.

The TaToo Web portal is in principle composed of a set of portlets that match with the set of services provided by the TaToo Semantic Framework: Tagging Portlets, Discovery Portlets, Evaluation and Validation Portlets. If a functionality requires a complex design, it can be provided throw the means of a set of the portlets..

The TaToo Web Portal interacts mainly with the portlets that make up the portal. The information exchanged between the portal and the portlets is not relevant at the specification level and depends on the specific portlet server / container.

\subsection{Tagging Portlet}

The Tagging Portlet interacts with the Tagging Service. It exchanges the following information with the Tagging Service:

- $\quad$ Domain selected by the user;

- $\quad$ Ontologies (in simplified representation) matching the domain;

- $\quad$ Tags (selected terms from an ontology);

- $\quad$ User context information (to associate a tag with a user).

The user accesses the TaToo Web Portal in tagging process and selects the Tagging Portlet user interface. The Tagging Portlet presents to the user one or more resources that were just discovered, or already known and stored. The user can add a new tag (meta-information). Once the user fills, for example, an input text box or chooses a term from a selection box on the Tagging Portlet, the meta-information entered is then sent to the Tagging Service associated with the selected resource(s). It will be then up to the TaToo Clearinghouse to manage storage of the metainformation associated with the resource(s). 


\subsection{Discovery Portlets}

There are three discovery portlets: Simple Search Portlet; Hierarchical Search Portlet; Result Presentation Portlet:

- $\quad$ Simple Search Portlet acts as a Web Client of the TaToo Discovery Service. To display the retrieved information, the Simple Search Portlet redirects the results to a TaToo User Component able to show results. This is the component responsible for the search and discovery of tagged environmental resources. It provides a GUI suitable for expressing the most relevant queries for a given domain. This component provides functionality to search and discover through the key concepts and relations chosen from the corresponding ontology domain. The user accesses the TaToo Web Portal in discovery process looking for search and discovery functionality. On the TaToo Web Portal the user can access the related Search and Discovery user interface. The Search and Discovery Portlet will contact the TaToo Service tier and in particular will access the Discovery Service, which will be responsible for forwarding the required operation to the Clearinghouse. The Simple Search Portlet asks the Discovery Service for Ontologies matching a domain selected by the user and sends the search terms (chosen from the ontology) to the Discovery Service. It receives search results from the Discovery Service in the form of URIs of matching resources along with some meta-information describing the resource.

- Hierarchical Search Portlet acts as a Web Client of the TaToo Discovery Service. To display the retrieved information, the Hierarchical Search Portlet redirects the search results to a TaToo User Component (e.g. the Result Presentation Portlet) able to show results. This is the component responsible for the search and discovery of tagged environmental resources. The Hierarchical Search Portlet provides a GUI suitable for the discovery strategy called navigation. It can be accessed by the user through its GUI which allows formulating the search query via a category tree. The user retrieves the resources belonging to a category (previously selected on the tree) and its related meta-information. The Hierarchical Search Portlet interacts with the TaToo Web Portal and the Discovery Service. It asks the Discovery Service for Ontologies matching a domain selected by the user and sends the search terms (chosen from the category tree) to the Discovery Service. It receives search results back in the form of URIs of matching resources along with some meta-information describing the resource.

- Result Presentation Portlet interacts with Search Portlets to present search results. It allows users to view, in a user-friendly manner, the results of a query, and to interact with them. To this end, the component will show different elements in different areas such as performed query, found resources, annotation related to those resources, and so on.

\subsection{TaToo Evaluate and Validate Portlet}

The TaToo Evaluate and Validate Portlet is a component responsible for the evaluation and validation of search results and tags associated with resources. It is 
part of the TaToo Presentation tier on the TaToo Web Portal providing evaluation and validation functionality to the user, communicating with the Evaluate and Validate Service.

It shows all available tags of a previously discovered resource including potentially attached validation meta-information. Validation meta-information, or validation tags, are special tags for the purpose of evaluation and validation. They are used, for example, to rate the quality, uncertainty, reliability, etc. of resources as well as tags (including Validation Tags). The portlet should also provide an indicator for overall level of quality, uncertainty, reliability, etc., of the resource computed from all available Validation Tags.

This Portlet interacts with the TaToo Web Portal, the Discovery Portlet, the Tagging Service and the Evaluate and Validate Service. It receives the URI of a resource from the Search Portlet receives associated Validation Tags from the Evaluate and Validate Service and sends new Validation Tags to the Evaluate and Validate Service.

\section{Conclusions}

In this paper we have introduced the aims and the vision of the MU scenario of FP7 project TaToo, which aims at providing a collaborative platform for the semantic enrichment of environmental information resources on the web. The main challenges of the TaToo project are the provision of an appealing user interface for the semantic annotation of environmental resources, more specifically environmental data, information, web services and models, and the development of a set of TaToo tools to provide a preliminary semantic analysis of the content of web resources, with the ability to access different published ontologies that describe the available knowledge basis.

Acknowledgments. The research leading to these results has received funding from the European Community's Seventh Framework Programme (FP7/2007-2013) under grant agreement nr. 247893.

\section{References}

1. Brabec, K., Jarkovský, J., Dušek, L., Kubásek, M., Hřebíček, J., Holoubek, I., Čupr, P., Klánová, J.: GENASIS: System for the Assessment of Environmental Contamination by Persistent Organic Pollutants. In: EnviroInfo 2009. Environmental Informatics and Industrial Environmental Protection: Concepts, Methods and Tools. 23. International Conference on Informatics for Environmental Protection, pp. 369-376. Shaker Verlag, Aachen (2009)

2. Dušek, L., Mužík, J., Koptíková, J., Brabec, P., Žaloudík, J., Vyzula, R., Kubásek, M.: The national web portal for cancer epidemiology in the Czech Republic. In: Enviroinfo 2005. 19th International Conference Informatics for Environmental Protection, pp. 434-439. Masaryk University Press, Brno (2005)

3. Dušek, L.: Czech Cancer Care in Numbers 2008-2009. Grada Publishing, Praha (2009) 
4. Hřebíček, J., Dušek, L., Kubásek, M., Jarkovský, J., Brabec, K., Holoubek, I., Kohút, L., Urbánek, J.: Anthropogenic Impact and Global Climate Change. Description of Validation Scenario in TaToo Project. In: 7. letní škola aplikované informatiky. Indikátory účinnosti EMS podle odvětví, pp. 6-23. nakladatelství Littera, Brno (2010)

5. Hřebíček, J., Dušek, L., Kubásek, M., Jarkovský, J., Brabec, K., Holoubek, I., Kohút, L., Urbánek, J.: Validation Scenario for Anthropogenic Impact and Global Climate Change for Tatoo. In: Proceedings of the Workshop "Environmental Information Systems and Services - Infrastructures and Platforms", Aachen. CEUR-WS (2010)

6. Klánová, J., Čupr, P., Holoubek, I., Borůvková, J., Přibylová, P., Kareš, R., Kohoutek, J., Dvorská, A., Komprda, J.: Towards the Global Monitoring of POPs - Contribution of the MONET Networks. In: RECETOX. Masaryk University, Brno (2009)

7. Pillmann, W., Hřebíček, J.: Information Sources for a European Integrated Environmental Information Space. In: EnviroInfo 2009. Environmental Informatics and Industrial Environmental Protection: Concepts, Methods and Tools. 23. International Conference on Informatics for Environmental Protection, pp. 341-352. Shaker Verlag, Aachen (2009)

8. Rizzoli, A., Schimak, G., Donatelli, M., Hřebíček, J., Avellino, G., Mon, J.: TaToo: tagging environmental resources on the web by semantic annotations. In: iEMSS 2010. International Congress on Environmental Modelling and Software. Modelling for Environment's Sake, pp. 1192-1199. iEMSS, Ottawa (2010)

9. Urbánek, J., Brabec, K., Dušek, L., Holoubek, I., Hřebíček, J., Kubásek, M.: Monitoring and assessment of environmental impact by persistent organic pollutants. In: Diamantaras, K., Duch, W., Iliadis, L.S. (eds.) ICANN 2010. LNCS, vol. 6354, pp. 483-488. Springer, Heidelberg (2010) 